OPEN ACCESS

Edited by:

Laurence Morel,

University of Florida, United States

Reviewed by:

Roberto González-Amaro,

Universidad Autónoma de San Luis

Potosí, Mexico

Natascha Köstlin-Gille,

University Children's Hospital

Tübingen, Germany

*Correspondence:

Zhimin Zhai

aydxyk@163.com

Specialty section:

This article was submitted to Autoimmune and Autoinflammatory

Disorders,

a section of the journal

Frontiers in Immunology

Received: 16 January 2019

Accepted: 13 May 2019

Published: 29 May 2019

Citation:

Wang Z, Zhu F, Wang J, Tao Q, Xu X, Wang $H$, Xiong $S$, Wang $Y$ and Zhai $Z$ (2019) Increased

CD14+HLA-DR ${ }^{-/ l o w}$ Myeloid-Derived Suppressor Cells Correlate With

Disease Severity in Systemic Lupus

Erythematosus Patients in an iNOS-Dependent Manner.

Front. Immunol. 10:1202.

doi: 10.3389/fimmu.2019.01202

\section{Increased CD14+HLA-DR-/low Myeloid-Derived Suppressor Cells Correlate With Disease Severity in Systemic Lupus Erythematosus Patients in an iNOS-Dependent Manner}

\author{
Zhitao Wang ${ }^{1}$, Fengfeng Zhu ${ }^{1}$, Jiyu Wang ${ }^{1}$, Qianshan Tao ${ }^{2}$, Xuanxuan $X u^{2}$, \\ Huiping Wang ${ }^{1}$, Shudao Xiong ${ }^{1}$, Yiping Wang ${ }^{3}$ and Zhimin Zhai ${ }^{1 *}$
}

${ }^{1}$ Department of Hematology, The Second Affiliated Hospital of Anhui Medical University, Hefei, China, ${ }^{2}$ Department of Rheumatology, The Second Affiliated Hospital of Anhui Medical University, Hefei, China, ${ }^{3}$ Centre for Transplantation and Renal Research, Westmead Millennium Institute, The University of Sydney, Sydney, NSW, Australia

Myeloid-derived suppressor cells (MDSCs) comprise of a population of cells, which suppress the innate and adaptive immune system via different mechanisms. MDSCs are accumulated under pathological conditions. The present study aimed to clarify the pathological role of MDSCs in systemic lupus erythematosus (SLE) patients. Consequently, the level of circulating M-MDSCs was significantly increased in newly diagnosed SLE patients as compared to healthy controls. An elevated level of M-MDSCs was positively correlated with the disease severity in SLE patients and an immunosuppressive role was exerted in an iNOS-dependent manner. The decrease in the number of M-MDSCs after therapy rendered them as an indicator for the efficacy of treatment. These results demonstrated that M-MDSCs participated in the pathological progress in SLE patients. Thus, MDSCs are attractive biomarkers and therapeutic targets for SLE patients.

Keywords: myeloid-derived suppressor cells, SLE, iNOS, disease severity, immunosuppression

\section{INTRODUCTION}

Systemic lupus erythematosus (SLE) is one of the most common autoimmune diseases (1). The main manifestations include the imbalance of peripheral immune tolerance to autoantigens. The excessive activation of $\mathrm{T}$ cells and the production of a large number of autoantibodies due to the hyperfunction of B cells disrupt the steady state of pro-inflammatory and anti-inflammatory factors as well as the accumulation of large amounts of immune complexes and inflammatory damage to multiple organs and tissue systems (2). The equilibrium in the immune system and its strict control by regulatory mechanisms is a critical issue in SLE. Interestingly, several individual cell subsets or molecules are involved in the pathogenesis of SLE. However, the specific pathogenesis is not yet clearly understood. 
Myeloid-derived suppressor cells (MDSCs) are a group of immature cells that can inhibit the immune response, thereby promoting the occurrence and progress of tumors as described previously (3). MDSCs can promote tumor metastasis and angiogenesis by inhibiting the immune response and $\mathrm{T}$ cell proliferation (4). Also, MDSCs express Gr-1 and CD11b surface molecular markers that are divided into two types: granulocytic and monocytic phenotypes $(5,6)$. Anti-Gr-1 monoclonal antibody contains two molecules, Ly6G and Ly6C. In mice, MDSCs are specified

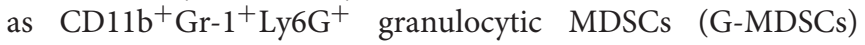

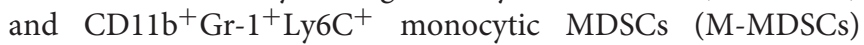
according to the phenotype $(7,8)$. Nonetheless, an unequivocal method for phenotyping the human MDSCs for the human equivalent of $\mathrm{Gr}-1$ is lacking. G-MDSCs are primarily

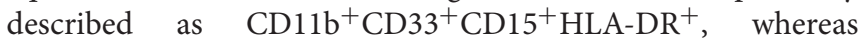
M-MDSCs are mainly described as $\mathrm{CD} 11 \mathrm{~b}^{+} \mathrm{CD} 33^{+} \mathrm{CD} 14^{+} \mathrm{HLA}-$ $\mathrm{DR}^{\text {low } /-}$ or $\mathrm{CD}^{+} 4^{+} \mathrm{HLA}-\mathrm{DR} \mathrm{R}^{\text {low/- }}$ in humans (9-11). MDSCs inhibit the immune response through different pathways, including direct and indirect contact. For example, MDSCs secrete a variety of immunomodulatory factors to exert an inhibitory effect, including inducible nitric oxide synthase (iNOS) and arginase-1 (Arg-1) (12, 13). Previous studies have shown that MDSCs can be accumulated by several cytokines in vitro, for example granulocyte macrophage colony stimulating factor (GM-CSF) and interleukin-6 (IL-6) (14).

In recent years, the role of MDSCs has attracted increasing attention with respect to autoimmune diseases (15). These cells are involved in the occurrence and development of a variety of autoimmune diseases, including rheumatoid arthritis (RA) (16), type I diabetes (TID) (17), multiple sclerosis (MS) (18), and inflammatory bowel disease (IBD) (19). Moreover, previous studies mainly focused on mouse models, and only a few studies were carried out in patients (2023). Previously, $\mathrm{Wu}$ et al. found active SLE patients had a significant increase in $\mathrm{HLA}_{-} \mathrm{DR}^{-} \mathrm{CD} 11 \mathrm{~b}^{+} \mathrm{CD} 33^{+}$MDSCs, including both $\mathrm{CD} 14^{+} \mathrm{CD} 6 \mathrm{~b}^{-}$monocytic and $\mathrm{CD} 14^{-} \mathrm{CD}^{-} 6 \mathrm{~b}^{+}$ granulocytic MDSCs in the peripheral blood as compared to healthy controls (24). The frequency of M-MDSCs was positively correlated with disease severity and MDSCs were pathogenic for SLE by induction of Th17 cells. However, the role of MDSCs in the pathogenesis of SLE needs further investigation.

In this study, we evaluated the correlation of another


with clinical parameters and the possible mechanisms in pathogenesis of SLE patients. We found that the level of circulating M-MDSCs was significantly increased and positively correlated with the disease activity in newly diagnosed SLE patients. Additionally, the elevated level of M-MDSCs exerted an immunosuppressive role in an iNOS-dependent manner. The current study provides a new theoretical basis for the pathogenic role of MDSCs in SLE and puts forth new therapeutic targets for the clinical treatment of SLE patients.

\section{MATERIALS AND METHODS}

\section{Patients and Samples}

A total of 32 patients, diagnosed with SLE from May 2016 to March 2018 at the Second Affiliated Hospital, Anhui Medical University, China, were enrolled in this study. Patients with comorbidities that might affect their immune status with respect to inflammation or tumors were excluded from the study. The detailed clinical data of the patients are shown in Table 1. All patients with SLE fulfilled the revised disease criteria of the American College of Rheumatology (25). The disease activity was assessed based on the SLE Disease Activity Index (SLEDAI) (26). According to the SLEDAI score, SLE patients were divided into two groups: active group $(n=18$, SLEDAI $>9)$ and inactive group $(n=14$, SLEDAI $\leq 9)$. The 32 newly diagnosed SLE patients were administered glucocorticoid, cyclophosphamide, or methotrexate therapy according to the patient's condition. The samples from these patients were collected prior to any treatment and after at least 3 months of treatment. Moreover, the samples were evaluated within $6 \mathrm{~h}$ of collection.

The study protocol was approved by the Ethics Committee of Anhui Medical University. Written informed consents were obtained from all patients and volunteers.

\section{Flow Cytometric Analysis}

The following monoclonal antibodies were purchased from Beckman Coulter Immunology (Miami, FL, USA): APC-labeled CD14 (clone RMO52), ECD-labeled HLA-DR (clone Immu375), FITC-labeled CD14 (clone 116), PE-labeled HLA-DR (clone B8.12.2), and PE-labeled anti-CD4 (clone 13B8.2). Peripheral blood mononuclear cells (PBMCs) were stratified on FicollHypaque (Amersham Biosciences, Sweden) and separated by centrifugation for $25 \mathrm{~min}(500 \times \mathrm{g})$. Subsequently, PBMCs were collected and washed with phosphate-buffered saline (PBS). After washing, $100 \mu \mathrm{L}$ PBMCs was incubated with $\mathrm{CD}$ antibody and analyzed by flow cytometry (FC500 MPL, Beckman Coulter), and EXPO 32 Multicomp software was used for data acquisition

TABLE 1 | Characteristics of healthy donors and SLE patients.

\begin{tabular}{lcc}
\hline State of disease at sample draw & No. of patients & Average age (range) \\
\hline $\begin{array}{l}\text { Newly diagnosed } \\
\text { Gender }\end{array}$ & 32 & $27.7(18-36)$ \\
$\quad$ Male & 6 & $28.6(26-33)$ \\
$\quad$ Female & 26 & $27.5(18-36)$ \\
Lupus nephritis & & \\
$\quad$ YES & 12 & $27.8(19-36)$ \\
NO & 20 & $27.7(18-33)$ \\
Active disease & & \\
$\quad$ YES & 18 & $27.2(18-36)$ \\
$\quad$ NO & 14 & $28(21-34)$ \\
Anti-dsDNA antibody & & \\
$\quad$ Positive & 20 & $29.5(20-36)$ \\
Negative & 12 & $24.8(18-32)$
\end{tabular}


and analysis. All the samples were matched with the same type of antibody as a control.

\section{Identification of MDSCs}

PBMCs were separated from 5 newly diagnosed SLE patients $($ SLEDAI > 10) and stained with antibodies to human CD4, CD14, and HLA-DR. In suppressive assays, autologous $\mathrm{CD} 4^{+} \mathrm{T}$

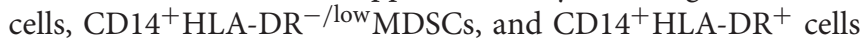
were sorted using MoFlo XDP cell sorter (Beckman Coulter). The purity of the cells was $>95 \%$ after sorting. $\mathrm{CD}^{+} \mathrm{T}$ cells were labeled with carboxy fluorescein succinimidyl ester (CFSE) (0.5 $\mu \mathrm{mol} / \mathrm{L}$ ) according to the manufacturer's instructions (Invitrogen, Carlsbad, CA, USA). CFSE-labeled $\mathrm{CD}^{+}{ }^{+} \mathrm{T}$ cells were washed and co-cultured with M-MDSCs and CD14 ${ }^{+}$HLA$\mathrm{DR}^{+}$cells in a ratio of 1:1 in 96-well plates (Wuxi Nest Biotechnology Co., Ltd, Wuxi, China) in complete RPMI 1640 medium with $10 \%$ fetal bovine serum (Gibco, Carlsbad, CA, USA). $\mathrm{CD}^{+} \mathrm{T}$ cells were cultured separately and used as positive control. All cells were incubated with anti-CD3 $(2 \mu \mathrm{g} / \mathrm{mL})$, anti-CD28 $(5 \mu \mathrm{g} / \mathrm{mL})$. Following 3 days of co-culture, the rate of proliferation of $\mathrm{CD}^{+} \mathrm{T}$ cells was determined by FACS analysis. The concentration of IFN- $\gamma$ was measured by ELISA Kit (R\&D systems, Minneapolis, MN, USA) according to the manufacturer's instructions.

\section{RNA Isolation and RT-PCR}

Total RNA was extracted from PBMCs using TRIzol reagent (Invitrogen). cDNA was synthesized by reverse transcription using oligo(dT). RT-PCR for $i N O S$ and a reference gene ( $\beta$-actin) was performed in the real-time PCR model 7300 (Applied Biosystems, USA) using Power SYBR Green PCR Master Mix (Applied Biosystems) according to the manufacturer's instructions. The expression of the target gene was normalized against that of $\beta$-actin. The primer sequences used were as follows: (5'-CTTTCCAAGACACACTTCACCA- $\left.3^{\prime}\right)$ and reverse (5'-TATCTCCTTTGTTAC CGCTTCC-3') for iNOS; forward (5'-TGGCACCCAGCACAATGAA- $\left.3^{\prime}\right)$ and reverse (5'-CTAAGTCATAGTCCGCT AGAAGCA- $\left.3^{\prime}\right)$ for $\beta$-actin.

\section{ELISA Assay}

Plasma was collected at the same time when PBMCs were isolated from SLE patients and healthy controls. Plasma concentrations of IL-6, GM-CSF, and Arg-1 were measured by ELISA Kit (R\&D systems).

\section{Cell Culture and Cytokine Induction}

PBMCs $\left(1 \times 10^{6}\right)$ from healthy controls were incubated with plasma $(500 \mu \mathrm{L})$ from 5 newly diagnosed SLE patients (SLEDAI $>10$ ) and 5 healthy controls in 24-well-plates in triplicate (Wuxi Nest Biotechnology) for $72 \mathrm{~h}$. Recombinant human granulocytemacrophage colony stimulating factor (rhGM-CSF, $10 \mathrm{ng} / \mathrm{mL}$; Sigma) was added to the mixture to enhance cell viability $(14,27)$. The cells were cultured at $37^{\circ} \mathrm{C}$ in a humidified $\mathrm{CO}_{2}$ incubator.

\section{Cell Isolation and Sorting}

After $72 \mathrm{~h}$, all cells were collected from PBMC cultures. Autologous $\mathrm{CD}^{+} \mathrm{T}$ Cells, CD $14^{+} \mathrm{HLA}_{-\mathrm{DR}}{ }^{-/ \mathrm{low}^{\mathrm{M}} \mathrm{MDS} \text {, and }}$ $\mathrm{CD} 14^{+} \mathrm{HLA}_{-} \mathrm{DR}^{+}$cells were sorted as described above.

\section{Assay for T Cell Proliferation}

$\mathrm{CD}^{+}{ }^{+} \mathrm{T}$ cells were labeled with $0.5 \mu \mathrm{mol} / \mathrm{L}$ CFSE. Subsequently, the cells were washed and co-cultured at different ratios with M-MDSCs $(16: 1,4: 1,1: 1)$ and CD14 ${ }^{+}$HLA-DR $^{+}$cells $(1: 1)$ in 96-well plates in complete RPMI 1640 medium with $10 \%$ fetal bovine serum. $\mathrm{CD} 4^{+} \mathrm{T}$ cells cultured alone were used as positive control. All cells were incubated with anti-CD3 $(2 \mu \mathrm{g} / \mathrm{mL})$, antiCD28 $(5 \mu \mathrm{g} / \mathrm{mL})$. After 3 days of co-culture, the proliferation of $\mathrm{CD} 4^{+} \mathrm{T}$ cells was determined by FACS analysis. Additionally, the inhibitors of candidate suppressive molecules were added at the following final concentrations in the co-culture of $\mathrm{CD}^{+} \mathrm{T}$ cells and M-MDSCs (1:1): $500 \mu \mathrm{mol} / \mathrm{L} \mathrm{Nx-Hydroxy-nor-L-arginine,}$ diacetate salt (nor-NOHA, Calbiochem, Darmstadt, Germany), and $500 \mu \mathrm{mol} / \mathrm{L}$ NG-Methyl-L-arginine acetate salt (L-NMMA, Sigma-Aldrich). Supernatants were stored at $-80^{\circ} \mathrm{C}$ until further use. The concentration of IFN- $\gamma$ was measured by ELISA Kit according to the manufacturer's instructions.

\section{Statistical Analysis}

SPSS17.0 software was used for statistical analysis (SPSS Inc., Chicago, IL, USA). Paired and unpaired samples were tested for statistical significance by non-parametric Mann-Whitney $U$ test and parametric Student's $t$-test. Univariate analysis of variance (ANOVA) and LSD $t$-test were used to compare the levels of M-MDSCs in different groups. Pearson's coefficient test was employed for correlation analysis. In all the analyses, $P<0.05$ was considered significant.

\section{RESULTS}

\section{Expression of M-MDSCs in SLE Patients Is Increased}

Hitherto, there is no uniform standard for cell surface markers of human MDSCs. Although G-MDSCs are prevalent, the M-MDSCs exert a stronger immunosuppressive effect than G-MDSCs (28). Peripheral CD $14^{+} \mathrm{HLA}^{-\mathrm{DR}}{ }^{-/ \text {low }}$ cells were characterized as M-MDSCs in this study. We investigated the frequency of M-MDSCs in 32 newly diagnosed SLE patients, as well as 30 age- and sex-matched healthy controls. In $\mathrm{CD}_{14}{ }^{+}$monocytes and PBMCs, the frequency of M-MDSCs was significantly higher in SLE patients than that in healthy controls (Figures 1A-C). Next, the immunosuppressive ability of $\mathrm{CD}_{14}{ }^{+} \mathrm{HLA}_{-\mathrm{DR}}{ }^{-/ \text {low }}$ cells was investigated, which was the central element to identify MDSCs. Compared to the CD $14^{+} \mathrm{HLA}^{-\mathrm{DR}}{ }^{+}$cells, M-MDSCs significantly inhibited the proliferation of $\mathrm{CD}^{+} \mathrm{T}$ cells (Figure 1D). Moreover, the ability of $\mathrm{CD}^{+} \mathrm{T}$ cells co-cultured with M-MDSCs to produce IFN$\gamma$ was significantly decreased as compared to CD14 ${ }^{+} \mathrm{HLA}_{-} \mathrm{DR}^{+}$ cells (Figure 1E).

\section{Expansion of M-MDSCs Is Positively Correlated With Disease Severity and Response to Treatment in SLE Patients}

Further studies revealed that the frequency of M-MDSCs was corrected with the severity of the disease. In present study, we grouped all SLE patients by types of clinicopathological 
A

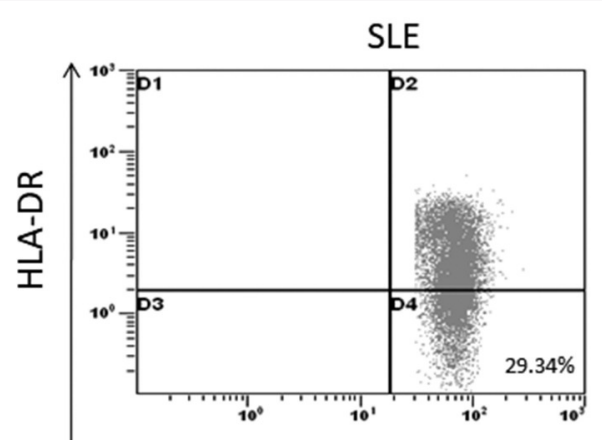

$\mathrm{HC}$

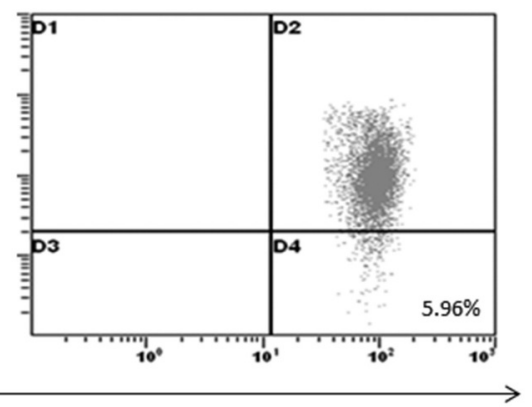

CD14
B

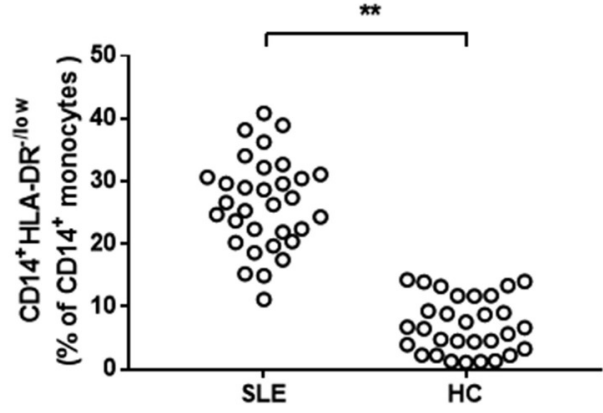

D

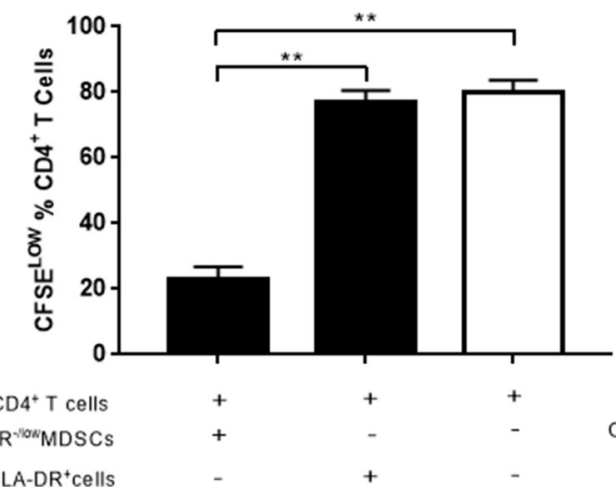

C



$\mathbf{E}$

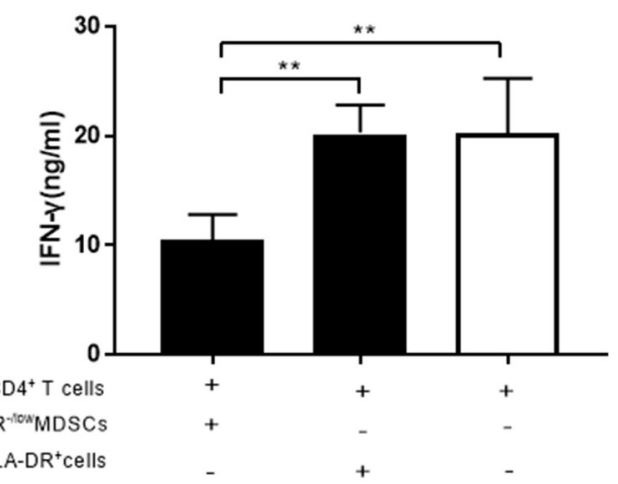

FIGURE 1 | The expression of M-MDSCs in SLE patients. (A) Representative flow cytometric speckle maps showed the expression of M-MDSCs in newly diagnosed SLE patients and healthy controls. (B) M-MDSCs levels in newly diagnosed SLE patients and healthy controls (in CD14+ monocytes). (C) M-MDSCs levels in newly diagnosed SLE patients and healthy controls (in PBMCs). (D) MDSCs had a significantly immunosuppressive activity on CD4+T cells as compared to the CD14 ${ }^{+} \mathrm{HLA}-\mathrm{DR}{ }^{+}$cells from active SLE patients. The proliferation of $\mathrm{CD} 4^{+} \mathrm{T}$ cells was measured by dilution of CFSE staining intensity using flow cytometry. (E) IFN- $\gamma$-producing was also significantly decreased in CD4+T cells co-cultured with M-MDSCs. Each point represents an individual. The horizontal bar represents the average. ${ }^{\star \star} P<0.01$.

factors, which indicated disease progression, including gender, lupus nephritis, anti-dsDNA antibody, and disease activity. Higher levels of M-MDSCs were found in female patients as compared to male patients (Figure 2A). The frequency of MMDSCs in patients with lupus nephritis is higher than that in patients with normal renal function (Figure 2B). However, no significant difference was found between anti-dsDNA positive and anti-dsDNA-negative groups (Figure 2C). The frequency of
M-MDSCs was significantly decreased as compared to the same patients before treatment (Figure 2D).

In addition, the correlation between M-MDSCs and SLEDAI was analyzed. We found that M-MDSCs levels were higher in the active group than in the inactive group (Figure 2E). Furthermore, the score of SLEDAI was positively correlated with the frequency of M-MDSCs $(r=0.547$, $P<0.01$, Figure 2F). 
A

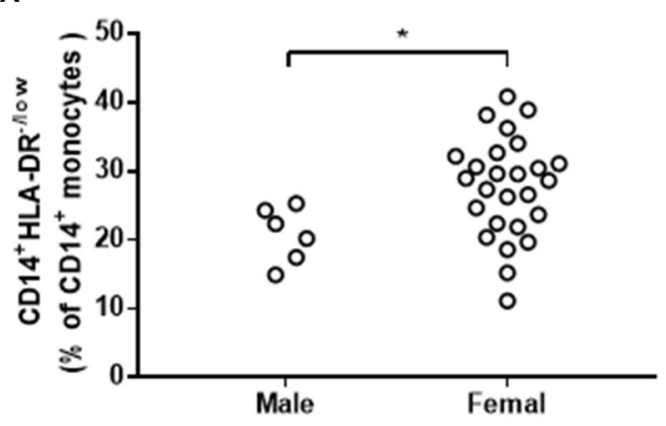

C

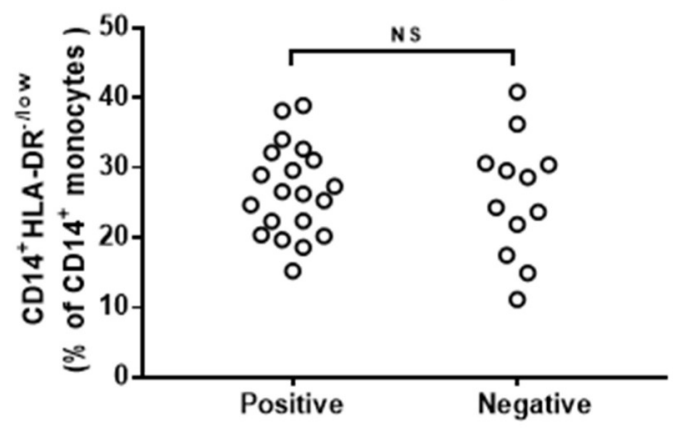

E

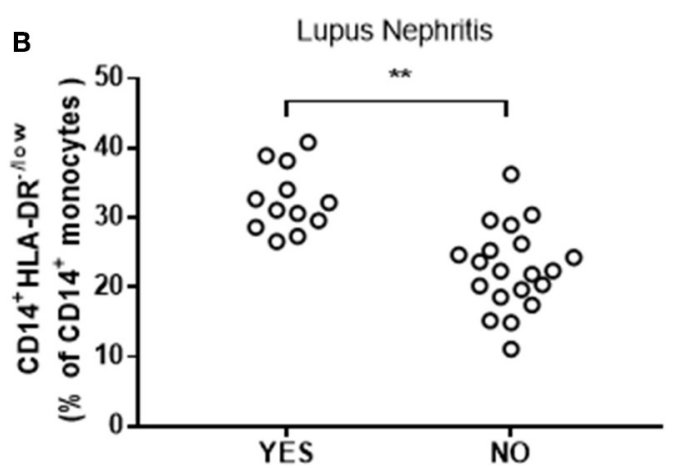

D



$\mathbf{F}$

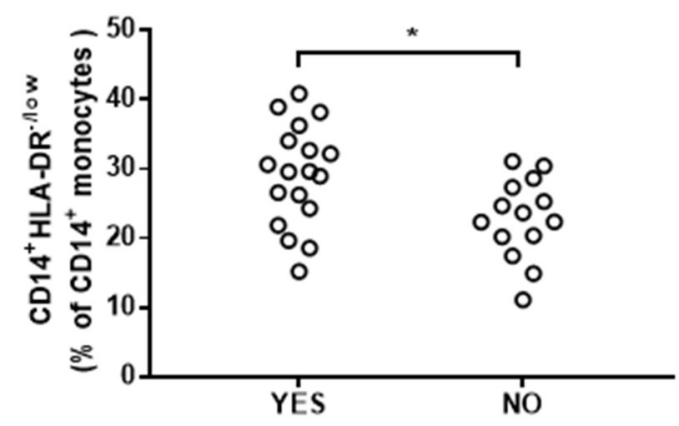

FIGURE 2 | Clinical correlation of M-MDSCs in SLE patients. (A) The frequency of M-MDSCs in female patients was significantly higher than that in male patients. (B) The frequency of M-MDSCs increased in patients with lupus nephritis. (C) No significant difference was detected in M-MDSCs between anti-dsDNA-positive and anti-dsDNA-negative groups (YES, lupus nephritis; NO, non-lupus nephritis). (D) The frequency of M-MDSCs was significantly decreased after treatment. (E) The level of M-MDSCs in patients with active SLE was higher than that in patients with inactive SLE (YES, active SLE patients; NO, inactive SLE patients). (F) M-MDSCs was positively correlated with the score of SLEDAl in SLE patients. Each point represents an individual. The horizontal bar represents the average. ${ }^{\star} P<0.05$; ${ }^{\star \star} P<0.01$.

\section{M-MDSCs-Associated Immunosuppressive Factors in SLE Patients}

M-MDSCs secrete a variety of cytokines and enzymes, including iNOS and Arg-1, to exert the immunosuppressive effect. Next, we detected the levels of MDSCs-associated enzymes in SLE patients. A significant increased level of iNOS mRNA was observed between SLE patients and healthy donors (Figure 3A). Also, a higher concentration of Arg-1 was found in SLE patients as compared to healthy donors, albeit not significantly (Figure 3B).

Previous studies have shown that M-MDSCs could be induced by specific cytokines, GM-SCF and IL-6, in the inflammatory state (14). Thus, we detected the levels of GM-SCF and IL-6 in the peripheral blood of SLE patients and found that they were significantly increased in the peripheral blood of newly diagnosed SLE patients (Figures 3C,D).

\section{Plasma From SLE Patients Induce the Expansion of M-MDSCs in vitro}

Furthermore, we determined the putative mechanisms of proliferation of M-MDSCs in vitro. Human PBMCs were isolated from 5 healthy volunteer controls and isolated by differential density gradient separation. An equivalent of $1 \times$ 




C

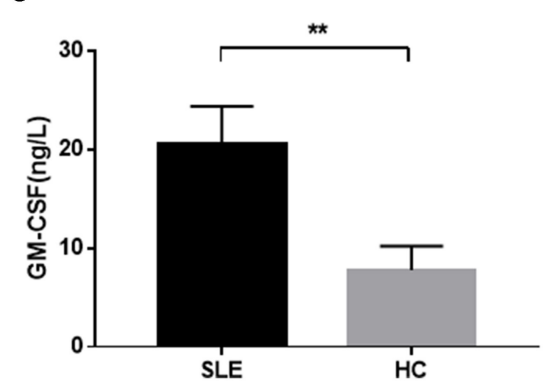

B

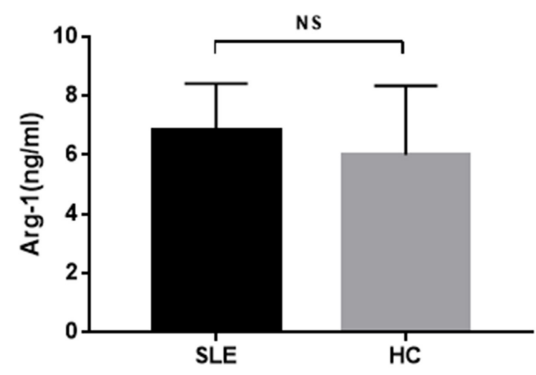

D

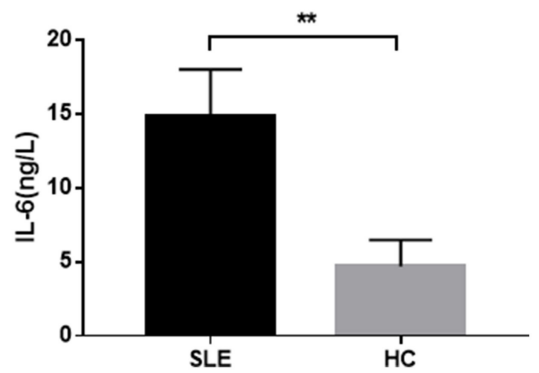

FIGURE 3 | The concentration of iNOS mRNA, Arg-1, GM-CSF, and IL-6 in newly diagnosed SLE patients. (A) The level of iNOS mRNA in SLE patients was significantly higher than that in healthy controls. (B) Plasma level of Arg-1 was not significantly elevated in SLE patients. (C,D) The plasma levels of GM-CSF and IL-6 in SLE patients were significantly higher than those in healthy controls. ${ }^{* \star} P<0.01$.

$10^{6}$ PBMCs from healthy controls was incubated with plasma $(500 \mu \mathrm{L})$ from newly diagnosed SLE patients (SLEDAI > 10) and healthy controls in 24-well plates for $72 \mathrm{~h}$ in vitro. As shown in Figures 4A,B, the percentage of M-MDSCs co-cultured with plasma from SLE patients was significantly increased as compared to the plasma from healthy controls as well as with pre-culture $(P<0.01)$.

\section{Immunosuppressive Effect of Induced M-MDSCs on Autologous CD4 ${ }^{+} \mathrm{T}$ Cells}

Firstly, the immunosuppressive activity of M-MDSCs on autologous $\mathrm{T}$ cell proliferation and IFN- $\gamma$ production was evaluated. Next, the suppression of $\mathrm{T}$ cell proliferation was assessed by CFSE dilution after a 3-days co-culture of MDSCs and compared to that elicited by HLA-DR ${ }^{+}$control cells. M-MDSCs significantly inhibited the proliferation of $\mathrm{CD} 4^{+} \mathrm{T}$ cells in a dosedependent manner as compared to the $\mathrm{CD} 14^{+} \mathrm{HLA}^{-\mathrm{DR}}{ }^{+}$cells (Figures 5A,C). Moreover, the ability of $\mathrm{CD} 4^{+} \mathrm{T}$ cells co-cultured with M-MDSCs to produce IFN- $\gamma$ was significantly decreased as compared to the $\mathrm{CD} 14^{+} \mathrm{HLA}_{-} \mathrm{DR}^{+}$cells (Figure $5 \mathrm{~B}$ ).

We found that the level of iNOS mRNA was significantly increased in SLE patients as compared to the healthy donors as described above. We hypothesized that M-MDSCs play an immunosuppressive role through iNOS in SLE patients. As we estimated, the proliferation of $\mathrm{CD}^{+} \mathrm{T}$ cells (Figure 5D) and production of IFN- $\gamma$ (Figure 5E) were significantly restored by L-NMMA, which is the specific inhibitor of iNOS. Moreover, this phenomenon was not found in the addition of Arg-1 inhibitor
Nor-NOHA, which further confirmed that the production of iNOS was the main method to exert an immunosuppressive effect on M-MDSCs in SLE patients (Figures 5D,E).

\section{DISCUSSION}

Myeloid suppressor cells are immature populations of immunosuppressive cells derived from bone marrow. Initially, the number of MDSCs expanded normally in the tumor, which inhibited the $\mathrm{T}$ cell immune response and promoted tumor progression (29). In recent years, the research field of MDSCs gradually involved with respect to autoimmune diseases. Accumulating evidence indicated that MDSCs participated in the inflammatory immune response to autoimmune diseases (30). Different from the role of MDSCs in promoting tumor progression, the role of MDSCs in autoimmune diseases is not clear. In several autoimmune disease mouse models, MDSCs increased significantly (31). However, MDSCs could not effectively alleviate the disease and also promote the progression of the disease. This contradicts the theory that MDSCs has immunosuppressive function and should inhibit the progression of autoimmune diseases, and hence, it role in autoimmune diseases needs to be further clarified.

In this study, we evaluated the impact of M-MDSCs on clinical parameters in SLE patients. As described above, validated unique phenotypic markers for MDSCs are yet lacking. The immunosuppressive effect is a critical feature in the identification of MDSCs (32). Firstly, the immunosuppressive 

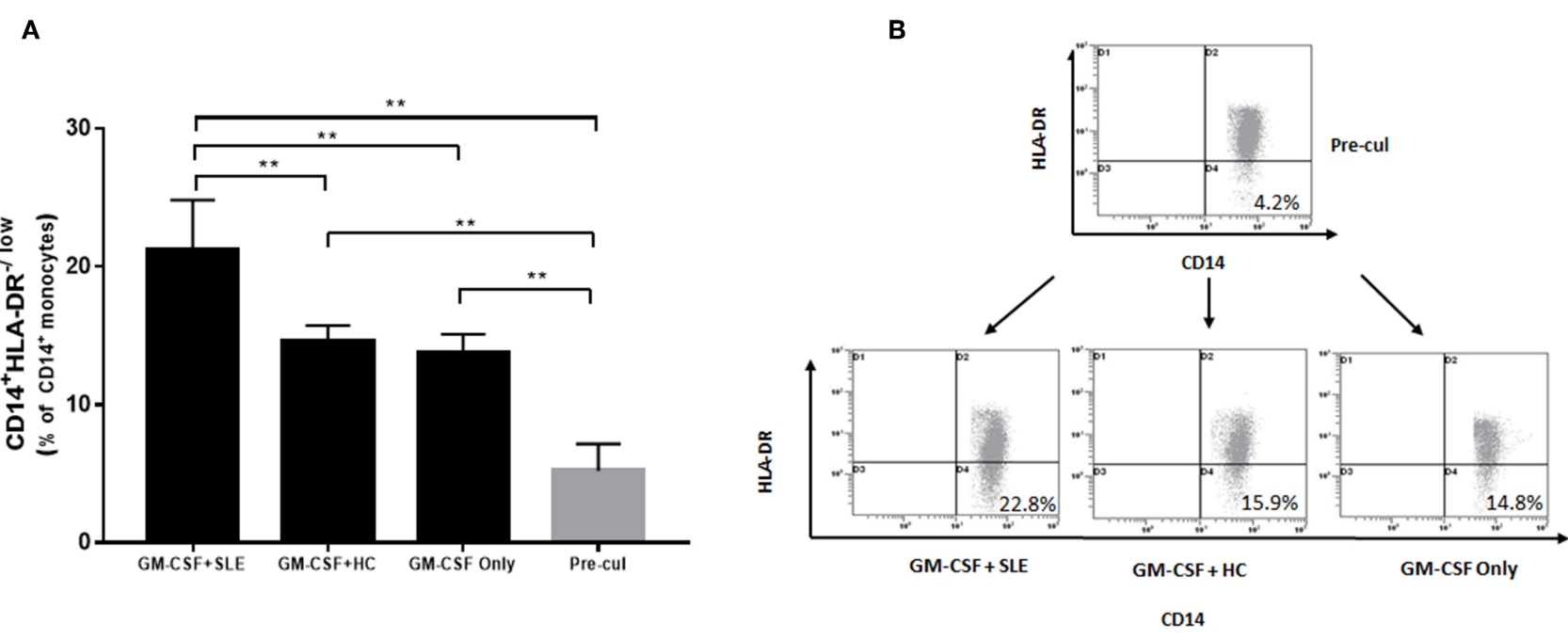

FIGURE 4 | Human PBMCs isolated from healthy controls were incubated with plasma from 5 newly diagnosed SLE patients and healthy controls. (A) Plasma from newly diagnosed SLE patients could induce a significant proliferation of M-MDSCs in vitro as compared to the plasma from healthy controls as well as with pre-culture $(P<0.01)$. (B) Representative flow cytometric dot plots showed the proliferation of M-MDSCs. Pre-cul, pre-culture; ${ }^{* \star} P<0.01$.

activity was investigated in $\mathrm{CD} 14^{+} \mathrm{HLA}-\mathrm{DR}{ }^{-/ \text {low }}$ cells from the peripheral blood of SLE patients, which was the central element to identify MDSCs. We evaluated the immunosuppressive activities of M-MDSCs on autologous $\mathrm{T}$ cell proliferation and IFN- $\gamma$ production. We found that M-MDSCs significantly suppressed the proliferation of $\mathrm{CD} 4^{+} \mathrm{T}$ cells as compared to the $\mathrm{CD} 14^{+} \mathrm{HLA}-\mathrm{DR}{ }^{+}$cells. Moreover, the production of IFN- $\gamma$ was significantly decreased in $\mathrm{CD} 4^{+} \mathrm{T}$ cells co-cultured with $\mathrm{M}$ MDSCs. Strikingly, M-MDSCs exert a strong suppressive ability on $\mathrm{T}$ cell proliferation and IFN- $\gamma$ production; however, this ability was not found in $\mathrm{CD} 14^{+} \mathrm{HLA}_{-} \mathrm{DR}^{+}$cells.

A significantly higher level of M-MDSCs was detected as compared to healthy controls. This phenomenon was in agreement with previous reports that MDSCs were involved in the pathological progress in SLE patients $(23,24)$. The frequency of M-MDSCs was increased markedly in female and lupus nephritis patients. Also, a positive correlation was established between the frequency of MDSCs and the disease activity in newly diagnosed SLE patients. Furthermore, the frequency of M-MDSCs decreased significantly after treatment. This observation indicated that the level of $\mathrm{M}$ MDSCs was correlated with disease severity. The decrease in the frequency of M-MDSCs after treatment was due to the reduction in disease activity. Consequently, significant changes in the frequency of M-MDSCs after treatment can be used to reflect the efficacy of treatment in SLE patients. This phenomenon indicated that MDSC expansion could be recognized as a major pathophysiological feature in human SLE patients.

MDSCs inhibit immune system through several different mechanisms (33). It is also speculated that MDSCs inhibit the immune cells by direct contact between cells or indirectly by inducing the production of Tregs and the secretion of a variety of cytokines (34); for example, iNOS, Arg-1, ROS, and IL-10.
G-MDSCs inhibit the $\mathrm{T}$ cell function by regulating ROS, while M-MDSCs inhibit the T cell function through iNOS and Arg$1(35,36)$. In the present study, the level of iNOS mRNA was significantly increased in newly diagnosed SLE patients. This indicated that MDSCs were an abundant source of iNOS production. MDSCs exerted immunosuppression in an iNOSdependent manner.

Tumor cells and tumor-derived factors (such as GM-CSF and IL-6) can induce the accumulation of MDSCs in PBMCs from healthy donors $(37,38)$. GM-CSF and IL-6 are vital proinflammatory cytokines in human immune regulation $(39,40)$. Interestingly, the plasma concentration of GM-CSF and IL-6 was significantly increased in newly diagnosed SLE patients. Next, we determined the possible mechanisms underlying the in vitro proliferation of M-MDSCs. Human PBMCs isolated from healthy volunteers were incubated with plasma from newly diagnosed SLE patients and healthy volunteer controls. The plasma from newly diagnosed SLE patients could induce the proliferation of MDSCs in vitro. This phenomenon suggested that immune tolerance abnormalities occurred in SLE patients. A large number of activated $\mathrm{T}$ and $\mathrm{B}$ cells secreted inflammatory cytokines, such as GM-CSF and IL-6. MDSCs could be induced by these inflammatory cytokines in SLE patients.

Furthermore, MDSCs are defined by surface markers in conjunction with suppressive function (32). The immunosuppressive activities and mechanisms of plasmainduced M-MDSCs were evaluated on autologous $\mathrm{T}$ cell proliferation and IFN- $\gamma$ production. M-MDSCs were found to significantly suppress the proliferation of $\mathrm{CD} 4^{+} \mathrm{T}$ cells in a dose-dependent manner as compared to the CD14 ${ }^{+} \mathrm{HLA}$ $\mathrm{DR}^{+}$cells. Moreover, IFN- $\gamma$-production was significantly decreased in $\mathrm{CD}^{+} \mathrm{T}$ cells co-cultured with M-MDSCs. Also, the proliferation of $\mathrm{T}$ cells and production of IFN- $\gamma$ were significantly restored by L-NMMA, which was the specific 
A

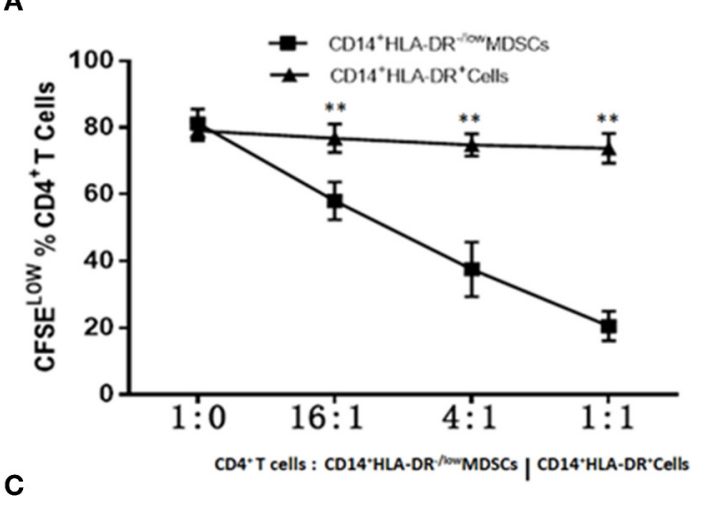

B

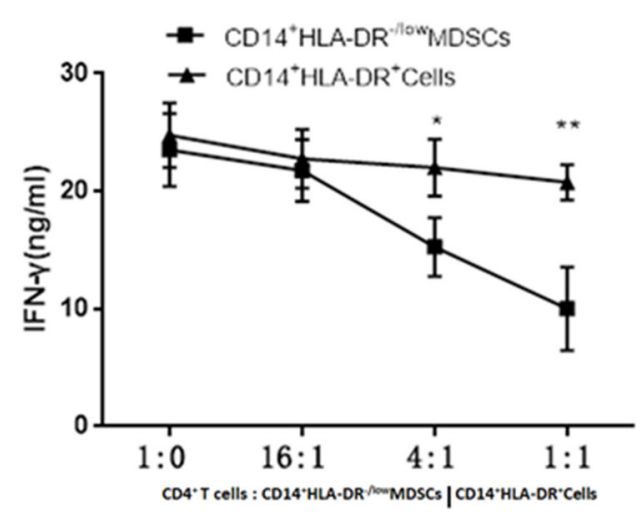


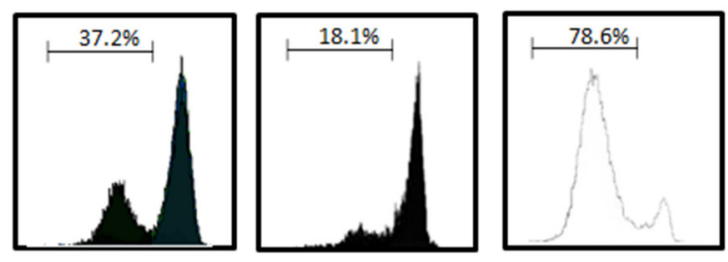

CFSE

1:0

16:1

$4: 1$

CD4 ${ }^{+} \mathrm{T}$ cells : CD14+HLA-DR-/low MDSCs

$1: 1$

$1: 1$ CD14 ${ }^{+}$HLA-DR ${ }^{+}$Cells

D

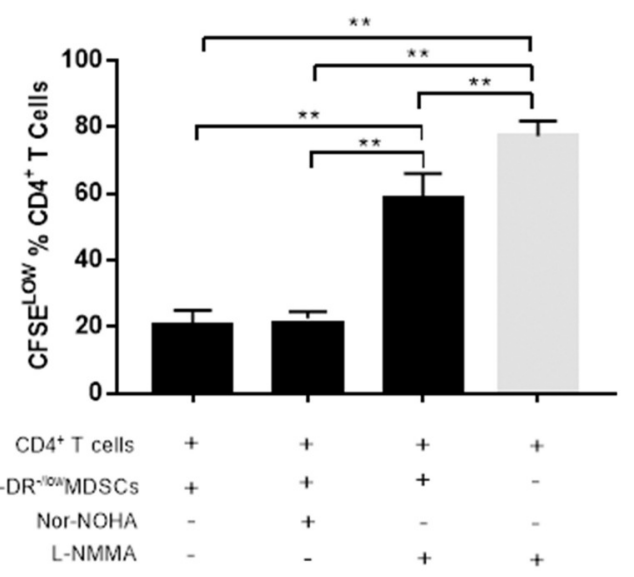

E

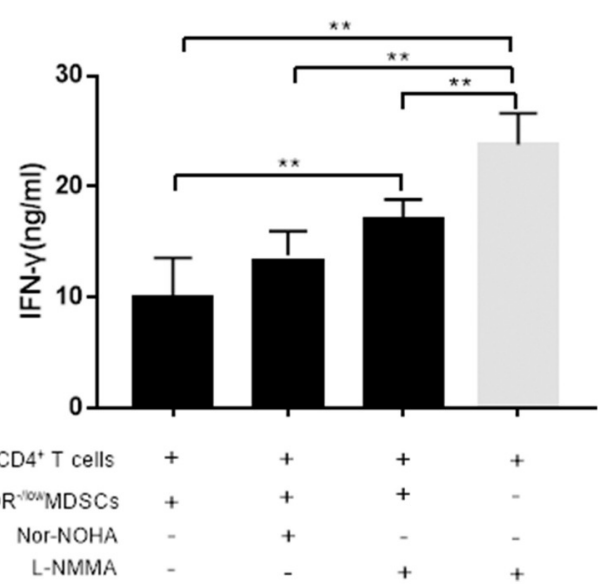

FIGURE 5 | Effects of M-MDSCs on the proliferation of autologous T cells and production of IFN- $\gamma$. (A) Plasma-induced MDSCs had a significant immunosuppressive activity on $\mathrm{CD}^{+}{ }^{+} \mathrm{T}$ cells in a dose-dependent manner as compared to the $\mathrm{CD} 14^{+} \mathrm{HLA}-\mathrm{DR}{ }^{+}$cells. The proliferation of $\mathrm{CD} 4^{+} \mathrm{T}$ cells was measured by dilution of $\mathrm{CFSE}$ staining intensity using flow cytometry. (B) IFN- $\gamma$-production was also significantly decreased in CD4 ${ }^{+} \mathrm{T}$ cells co-cultured with M-MDSCs. (C) Representative flow cytometry flow histograms demonstrated the immunosuppressive activity of M-MDSCs and CD14 ${ }^{+} \mathrm{HLA}-\mathrm{DR}^{+}$cells. (D,E) Effects of iNOS and Arg- 1 inhibitors on the M-MDSC suppression with respect to CD4+T cell proliferation and IFN- $\gamma$ production. Ctrl, contro/; ${ }^{\star} P<0.05 ;{ }^{\star \star} P<0.01$.

inhibitor of iNOS. M-MDSCs induced by plasma from SLE patients mediated a potent suppression of autologous $\mathrm{T}$ cell proliferation and IFN- $\gamma$ production. We hypothesized that the high expression of MDSCs exerts immunosuppressive effect and mediates the abnormal immune tolerance of SLE through iNOS pathway. However, suppressing the abnormal hyperactivity of immune function of SLE is not sufficient. The adoptive infusion of exogenous MDSCs might alleviate the condition of SLE patients (41).

In summary, elevated MDSCs were positively correlated with disease activity in SLE patients. MDSCs were pathogenic for SLE patients. The current data highlights that MDSCs are immunosuppressive in SLE patients in an iNOS-dependent manner. The number of M-MDSCs decreased after treatment, 
suggesting that these cells can be used as one of the indicators to judge the treatment effect. Briefly, MDSCs are critical immunosuppressor agents during SLE progression and might represent effective therapeutic targets for the treatment of SLE patients.

\section{AUTHOR CONTRIBUTIONS}

ZZ developed the experimental design. ZW wrote the manuscript and prepared the tables and figures. JW, HW, SX, and FZ wrote a part of the manuscript. XX and QT enrolled the patients. YW edited the manuscript. All authors reviewed the manuscript.

\section{REFERENCES}

1. Tsokos GC. Systemic lupus erythematosus. N Engl J Med. (2011) 365:2110-21. doi: 10.1056/NEJMra1100359

2. Wahren-Herlenius $M$, Dorner $T$. Immunopathogenic mechanisms of systemic autoimmune disease. Lancet. (2013) 382:819-31. doi: 10.1016/S0140-6736(13)60954-X

3. Pyzer AR, Cole L, Rosenblatt J, Avigan DE. Myeloid-derived suppressor cells as effectors of immune suppression in cancer. Int J Cancer. (2016) 139:1915-26. doi: 10.1002/ijc.30232

4. Marvel D, Gabrilovich DI. Myeloid-derived suppressor cells in the tumor microenvironment: expect the unexpected. J Clin Invest. (2015) 125:3356-64. doi: 10.1172/JCI80005

5. Wei C, Wang Y, Ma L, Wang X, Chi H, Zhang S, et al. Rapamycin nanomicelle ophthalmic solution reduces corneal allograft rejection by potentiating myeloid-derived suppressor cells' function. Front Immunol. (2018) 9:2283. doi: 10.3389/fimmu.2018.02283

6. Chang CJ, Yang YH, Chiu CJ, Lu LC, Liao CC, Liang CW, et al. Targeting tumor-infiltrating Ly6G(+) myeloid cells improves sorafenib efficacy in mouse orthotopic hepatocellular carcinoma. Int J Cancer. (2018) 142:1878-89. doi: 10.1002/ijc.31216

7. Gabrilovich DI, Nagaraj S. Myeloid-derived suppressor cells as regulators of the immune system. Nat Rev Immunol. (2009) 9:162-74. doi: 10.1038/nri2506

8. Tsiganov EN, Verbina EM, Radaeva TV, Sosunov VV, Kosmiadi GA, Nikitina IY, et al. Gr-1dimCD11b+ immature myeloid-derived suppressor cells but not neutrophils are markers of lethal tuberculosis infection in mice. J Immunol. (2014) 192:4718-27. doi: 10.4049/jimmunol.1301365

9. Manjili MH. Phenotypic plasticity of MDSC in cancers. Immunol Invest. (2012) 41:711-21. doi: 10.3109/08820139.2012.673670

10. Greten TF, Manns MP, Korangy F. Myeloid derived suppressor cells in human diseases. Int Immunopharmacol. (2011) 11:802-7. doi: 10.1016/j.intimp.2011.01.003

11. Zhao F, Hoechst B, Duffy A, Gamrekelashvili J, Fioravanti S, Manns MP, et al. S100A9 a new marker for monocytic human myeloid-derived suppressor cells. Immunology. (2012) 136:176-83. doi: 10.1111/j.1365-2567.2012. 03566.x

12. Talmadge JE, Gabrilovich DI. History of myeloid-derived suppressor cells. Nat Rev Cancer. (2013) 13:739-52. doi: 10.1038/nrc3581

13. Ohl K, Tenbrock K. Reactive oxygen species as regulators of MDSCmediated immune suppression. Front Immunol. (2018) 9:2499. doi: 10.3389/fimmu.2018.02499

14. Lechner MG, Liebertz DJ, Epstein AL. Characterization of cytokineinduced myeloid-derived suppressor cells from normal human peripheral blood mononuclear cells. J Immunol. (2010) 185:2273-84. doi: 10.4049/jimmunol.1000901

15. Li M, Zhu D, Wang T, Xia X, Tian J, Wang S. Roles of myeloid-derived suppressor cell subpopulations in autoimmune arthritis. Front Immunol. (2018) 9:2849. doi: 10.3389/fimmu.2018.02849

16. Fujii W, Ashihara E, Hirai H, Nagahara H, Kajitani N, Fujioka K, et al. Myeloid-derived suppressor cells play crucial roles in the regulation

\section{FUNDING}

This work was supported by the Natural Science Foundation of China (81670179) and Funding Program for the Cultivation of Academic and Technical Leaders in Colleges and Universities in 2014.

\section{ACKNOWLEDGMENTS}

We are grateful to the volunteers for participation in this study. of mouse collagen-induced arthritis. J Immunol. (2013) 191:1073-81. doi: 10.4049/jimmunol.1203535

17. Yin B, Ma G, Yen CY, Zhou Z, Wang GX, Divino CM, et al. Myeloid-derived suppressor cells prevent type 1 diabetes in murine models. J Immunol. (2010) 185:5828-34. doi: 10.4049/jimmunol.0903636

18. Guan Q, Moreno S, Qing G, Weiss CR, Lu L, Bernstein CN, et al. The role and potential therapeutic application of myeloid-derived suppressor cells in TNBS-induced colitis. J Leukoc Biol. (2013) 94:803-11. doi: $10.1189 / \mathrm{jlb} .0113050$

19. Ioannou M, Alissafi T, Lazaridis I, Deraos G, Matsoukas J, Gravanis A, et al. Crucial role of granulocytic myeloid-derived suppressor cells in the regulation of central nervous system autoimmune disease. J Immunol. (2012) 188:1136-46. doi: 10.4049/jimmunol.1101816

20. Iwata Y, Furuichi K, Kitagawa K, Hara A, Okumura T, Kokubo $\mathrm{S}$, et al. Involvement of CD11b+ GR-1 low cells in autoimmune disorder in MRL-Fas lpr mouse. Clin Exp Nephrol. (2010) 14:411-7. doi: 10.1007/s10157-010-0309-9

21. Trigunaite A, Khan A, Der E, Song A, Varikuti S, Jorgensen TN. Gr-1(high) $\mathrm{CD} 11 \mathrm{~b}+$ cells suppress B cell differentiation and lupus-like disease in lupusprone male mice. Arthritis Rheum. (2013) 65:2392-402. doi: 10.1002/art.38048

22. Der E, Dimo J, Trigunaite A, Jones J, Jorgensen TN. Gr1+ cells suppress T-dependent antibody responses in (NZB x NZW)F1 male mice through inhibition of $\mathrm{T}$ follicular helper cells and germinal center formation. $J$ Immunol. (2014) 192:1570-6. doi: 10.4049/jimmunol.1302479

23. Vlachou K, Mintzas K, Glymenaki M, Ioannou M, Papadaki G, Bertsias GK, et al. Elimination of granulocytic myeloid-derived suppressor cells in lupusprone mice linked to reactive oxygen species-dependent extracellular trap formation. Arthritis Rheumatol. (2016) 68:449-61. doi: 10.1002/art.39441

24. Wu H, Zhen Y, Ma Z, Li H, Yu J, Xu ZG, et al. Arginase-1-dependent promotion of TH17 differentiation and disease progression by MDSCs in systemic lupus erythematosus. Sci Transl Med. (2016) 8:331ra40. doi: 10.1126/scitranslmed.aae0482

25. Tan EM, Cohen AS, Fries JF, Masi AT, McShane DJ, Rothfield NF, et al. The 1982 revised criteria for the classification of systemic lupus erythematosus. Arthritis Rheum. (1982) 25:1271-7. doi: 10.1002/art.1780251101

26. Petri M. Disease activity assessment in SLE: do we have the right instruments? Ann Rheum Dis. (2007) 66 (Suppl. 3):iii61-4. doi: 10.1136/ard.2007.078477

27. Bayne LJ, Beatty GL, Jhala N, Clark CE, Rhim AD, Stanger BZ, et al. Tumorderived granulocyte-macrophage colony-stimulating factor regulates myeloid inflammation and T cell immunity in pancreatic cancer. Cancer Cell. (2012) 21:822-35. doi: 10.1016/j.ccr.2012.04.025

28. Haverkamp JM, Smith AM, Weinlich R, Dillon CP, Qualls JE, Neale $\mathrm{G}$, et al. Myeloid-derived suppressor activity is mediated by monocytic lineages maintained by continuous inhibition of extrinsic and intrinsic death pathways. Immunity. (2014) 41:947-59. doi: 10.1016/j.immuni.2014.10.020

29. Kumar V, Patel S, Tcyganov E, Gabrilovich DI. The nature of myeloid-derived suppressor cells in the tumor microenvironment. Trends Immunol. (2016) 37:208-20. doi: 10.1016/j.it.2016.01.004

30. Crook KR, Liu P. Role of myeloid-derived suppressor cells in autoimmune disease. World J Immunol. (2014) 4:26-33. doi: 10.5411/wji.v4.i1.26 
31. Crook KR, Jin M, Weeks MF, Rampersad RR, Baldi RM, Glekas AS, et al. Myeloid-derived suppressor cells regulate $\mathrm{T}$ cell and B cell responses during autoimmune disease. J Leukoc Biol. (2015) 97:573-82. doi: 10.1189/jlb.4A0314-139R

32. Bronte V, Brandau S, Chen SH, Colombo MP, Frey AB, Greten $\mathrm{TF}$, et al. Recommendations for myeloid-derived suppressor cell nomenclature and characterization standards. Nat Commun. (2016) 7:12150. doi: $10.1038 /$ ncomms 12150

33. Umansky V, Blattner C, Fleming V, Hu X, Gebhardt C, Altevogt $\mathrm{P}$, et al. Myeloid-derived suppressor cells and tumor escape from immune surveillance. Semin Immunopathol. (2016) 39:295-305. doi: 10.1007/s00281-016-0597-6

34. Umansky V, Blattner C, Gebhardt C, Utikal J. The role of myeloid-derived suppressor cells (MDSC) in cancer progression. Vaccines. (2016) 4:E36. doi: $10.3390 /$ vaccines4040036

35. Motallebnezhad M, Jadidi-Niaragh F, Qamsari ES, Bagheri S, Gharibi T, Yousefi M. The immunobiology of myeloid-derived suppressor cells in cancer. Tumour Biol. (2016) 37:1387-406. doi: 10.1007/s13277-0154477-9

36. Rastad JL, Green WR. Myeloid-derived suppressor cells in murine AIDS inhibit B-cell responses in part via soluble mediators including reactive oxygen and nitrogen species, and TGF-beta. Virology. (2016) 499:9-22. doi: 10.1016/j.virol.2016.08.031

37. Gorgun GT, Whitehill G, Anderson JL, Hideshima T, Maguire C, Laubach J, et al. Tumor-promoting immune-suppressive myeloidderived suppressor cells in the multiple myeloma microenvironment in humans. Blood. (2013) 121:2975-87. doi: 10.1182/blood-2012-08448548

38. Wu CT, Hsieh CC, Lin CC, Chen WC, Hong JH, Chen MF. Significance of IL-6 in the transition of hormone-resistant prostate cancer and the induction of myeloid-derived suppressor cells. J Mol Med. (2012) 90:1343-55. doi: 10.1007/s00109-012-0916-x

39. Schafer ZT, Brugge JS. IL-6 involvement in epithelial cancers. J Clin Invest. (2007) 117:3660-3. doi: 10.1172/JCI34237

40. Casella G, Finardi A, Descamps H, Colombo F, Maiorino C, Ruffini F, et al. IL27, but not IL-35, inhibits neuroinflammation through modulating GM-CSF expression. Sci Rep. (2017) 7:16547. doi: 10.1038/s41598-017-16702-w

41. Guo C, Hu F, Yi H, Feng Z, Li C, Shi L, et al. Myeloid-derived suppressor cells have a proinflammatory role in the pathogenesis of autoimmune arthritis. Ann Rheum Dis. (2016) 75:278-85. doi: 10.1136/annrheumdis-2014-205508

Conflict of Interest Statement: The authors declare that the research was conducted in the absence of any commercial or financial relationships that could be construed as a potential conflict of interest.

Copyright (c) 2019 Wang, Zhu, Wang, Tao, Xu, Wang, Xiong, Wang and Zhai. This is an open-access article distributed under the terms of the Creative Commons Attribution License (CC BY). The use, distribution or reproduction in other forums is permitted, provided the original author(s) and the copyright owner(s) are credited and that the original publication in this journal is cited, in accordance with accepted academic practice. No use, distribution or reproduction is permitted which does not comply with these terms. 